\title{
L'intégration dans le tissu urbain des retenues d'orages
}

\author{
M. Frésiers*, M. Bourgogne** \\ * Lyonnaise des Eaux - Dumez - 91, rue Paulin, 33029 Bordeaux Cedex \\ ** Communauté urbaine de Bordeaux, Esplanade Charles de Gaulle - 33076 Bordeaux Cedex
}

L'agglomération bordelaise dispose d'un important réseau d'évacuation des eaux pluviales comprenant notamment 27 bassins d'orage d'une capacité totale de stockage de $1200000 \mathrm{~m}^{3}$.

Ces bassins, destinés à protéger des zones urbanisées du risque d'inondations, sont, dans leur grande majorité, construits au sein-même du tissu urbain. Il doivent donc, au-delà de la fonction de protection, répondre au besoin d'intégration dans leur environnement, besoin auquel la collectivité dans son ensemble, élus et population, est de plus en plus sensible.

Compte tenu de la densité du milieu urbain, plusieurs types de bassins peuvent être conçus, bassins enterrés ou bassins à l'air libre (bassins à sec ou à plan d'eau permanent) pour lesquels les conditions d'intégration sont spécifiques.

\section{Les bassins à plan d'eau permanent}

Lorsque les surfaces libres sont suffisantes et que la vocation de la zone le permet, les bassins de retenue peuvent prendre la forme de bassin à sec ou à plan d'eau permanent.

Ces deux types de bassins sont présents à Bordeaux et permettent d'offrir un espace paysagé accessible au public.

Cependant, le bassin à plan d'eau permanent apporte très certainement la meilleure solution d'intégration dans le milieu urbain car la fonction hydraulique, loin d'être dissimulée, constitue au contraire l'attrait principal de la zone aménagée.

\section{L'expérience de Bordeaux}

Le premier bassin de ce type a été mis en service en 1969. Il s'agit du bassin de Bourran d'une capacité de stockage de $115000 \mathrm{~m}^{3}$ réalisé par aménagement d'un plan d'eau existant au sein d'un parc ouvert au public et à forte valeur botanique.

Le débit de temps sec circule dans la canalisation de déviation $\varnothing 1000$, le bassin étant alimenté par surverse en période d'orage.

1. Schéma hydraulique du bassin de Bourran.

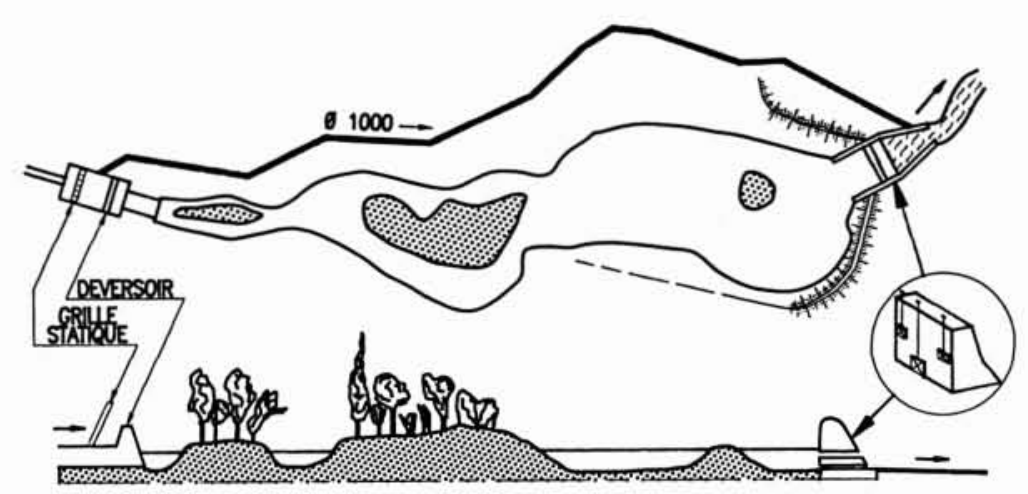


Depuis cette date, plusieurs bassins de ce type ont été réalisés. Parmi ceux-ci, deux bassins présentent des conditions d'activités particulières :

- le bassin de Fontaudin : Réalisation 1985 - Capacité $48000 \mathrm{~m}^{3}$. Existence d'une association de riverains développant des activités de loisirs et de pêche ;

- le bassin de Beaudesert : Réalisation 1991 - Capacité $69000 \mathrm{~m}^{3}$. Abords et plan d'eau aménagés en practice de golf.

Au total, 7 bassins à plan d'eau permanent sont gérés sur le territoire de la Communauté Urbaine représentant $30 \%$ de la capacité totale de stockage.

\section{Les contraintes spécifiques}

Ce type de bassin qui constitue un lieu de promenade et de loisir pour le public amène l'exploitant à faire face à de nouvelles contraintes liées au maintien de la qualité des eaux du bassin et au respect de l'écosystème qui s'y crée.

En effet, chaque orage apporte, outre une charge hydraulique, un flux de pollution organique et minérale dans un milieu récepteur restreint.

Lorsque les apports en pollution sont trop importants, le plan d'eau peut ne pas assurer une auto-épuration suffisante et une accumulation de vases se développe au fond du bassin avec risques de nuisances olfactives.

L'exploitant doit se transformer en gestionnaire d'écosystème et compléter son approche hydraulique par une approche écologique en contrôlant l'évolution des espèces végétales (roseaux, nénuphars, algues) et des espèces animales (poissons et oiseaux aquatiques).

Il convient donc de prévoir lors de la conception du profil du bassin des zones réservées aux végétaux et à la faune piscicole afin de développer la capacité d'autoépuration du bassin.

La figure 2 propose un exemple d'aménagement.

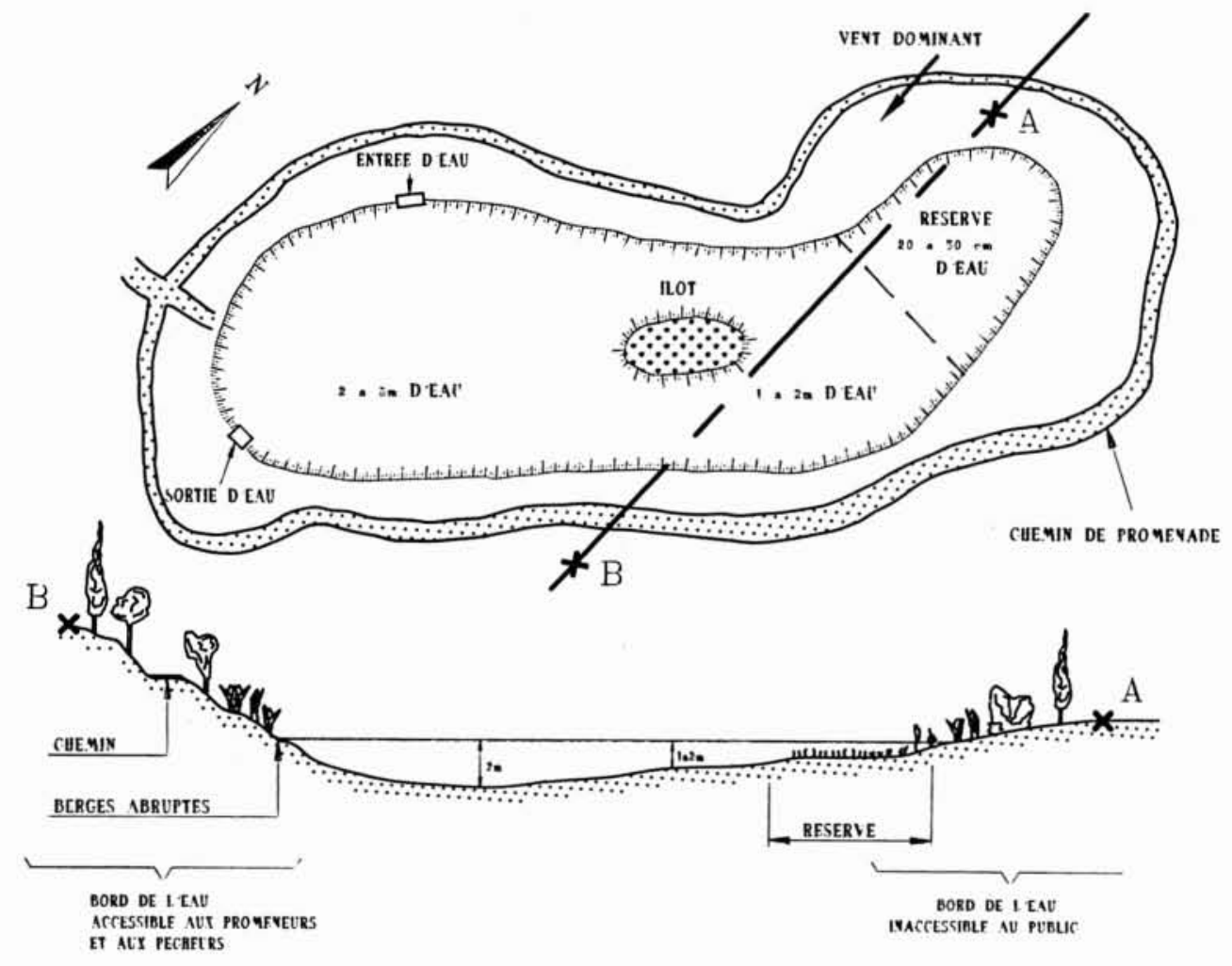

2. 


\section{Les bassins enterrés}

La rareté des espaces libres et le coût foncier orientent de fait les choix techniques vers la solution des bassins enterrés.

Leur existence laisse une large possibilité d'aménagement en surface (terrain de sport, bâtiment, parking...) et l'intégration de l'ouvrage en tant que tel ne soulève pas de problèmes de la part des riverains.

\section{L'expérience de Bordeaux}

L'expérience de la gestion de ce type de bassin remonte à sept ans et concerne un ensemble de 3 bassins :

\begin{tabular}{|l|c|c|}
\hline Désignation & $\begin{array}{c}\text { Capacité } \\
\text { de } \\
\text { stockage }\end{array}$ & $\begin{array}{c}\text { Année } \\
\text { de } \\
\text { réalisation }\end{array}$ \\
\hline PERINOT & $39000 \mathrm{~m}^{3}$ & 1984 \\
MAGINOT & $25000 \mathrm{~m}^{3}$ & 1985 \\
MIGRON BARDENNE & $8500 \mathrm{~m}^{3}$ & 1985 \\
\hline
\end{tabular}

Ces bassins sont construits sur le principe d'un remplissage et d'une vidange gravitaires. Ils sont séparés en compartiments avec seuils de déversement progressifs. Ce principe impose un stockage sur une faible hauteur et c'est donc la surface qui conditionne le volume à stocker. Il conduit également à définir des pentes faibles sous peine d'amputer ce volume (fig. 3).

Cette conception assure un fonctionnement fiable et nécessite des dépenses courantes d'entretien faibles. En revanche, elle rend les opérations de nettoyage du fond de bassin difficiles en raison de la surface importante à nettoyer $\left(10000 \mathrm{~m}^{2}\right.$ pour PERINOT) et de l'absence de pentes motrices pour la collecte des boues.

\section{Les contraintes spécifiques}

L'existence de ce type de bassin n'apparaît pas aux yeux du public et même des plus proches riverains.

Les nuisances envisagées lors des premiers projets du fait de l'existence d'un volume important en contact avec le réseau (mauvaises odeurs, insectes) ne sont pas apparues.

Les contraintes réelles proviennent des conditions d'évacuation des dépôts en fond de bassin qui se forment après chaque remplissage.

Cette opération nécessite la présence en surface de véhicules d'aspiration et de pompage pendant plusieurs jours entraînant des nuisances sonores importantes. Pour les nouveaux projets de bassins enterrés, la fonction décantation des eaux pluviales a été intégrée dans la conception même de l'ouvrage.

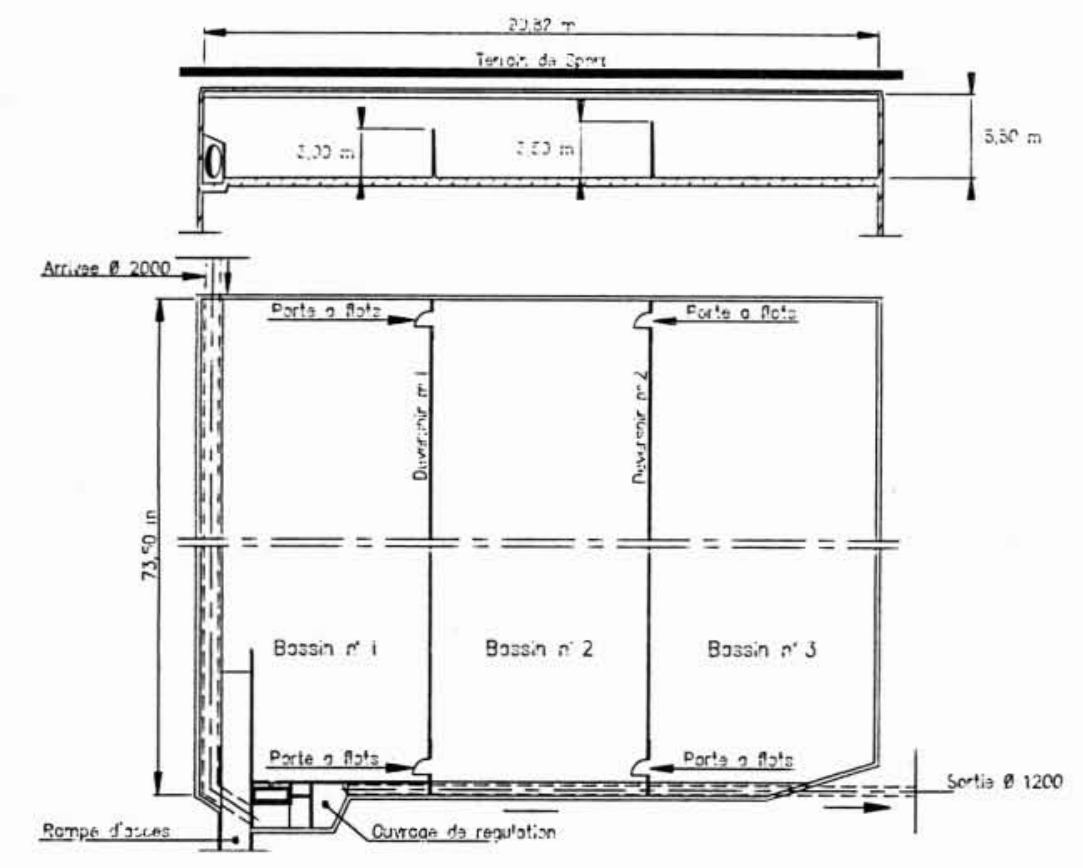

3. 


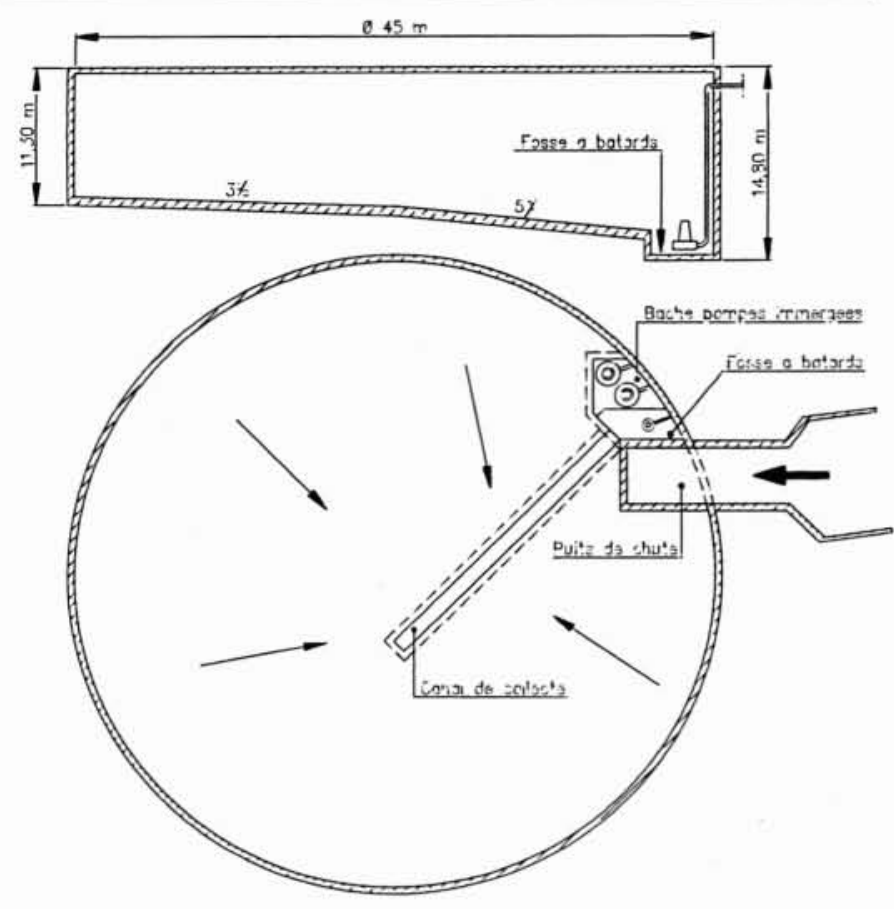

4.

Cette étude a conduit à une refonte complète de l'ouvrage enterré qui sera conçu sur le principe d'un remplissage gravitaire et d'une vidange par pompage.

Le bassin prendra la forme d'un ouvrage circulaire dont la profondeur dépendra du volume à stocker avec une pente suffisante pour collecter les boues vers une fosse de pompage. Le principe est illustré dans la figure 4 .

\section{Conclusion}

L'expérience de la gestion d'un ensemble de 27 bassins nous montre qu'une intégration réussie d'une retenue d'orage dans le tissu urbain doit passer par une démarche d'étude méthodologique et globale.

Les efforts réalisés ces dernières années par l'architecte et le technicien ont permis de réussir l'intégration physique de ces ouvrages.

L'enjeu devient alors de réussir l'intégration fonctionnelle des ouvrages. Afin de pérenniser cette intégration au fil du temps, des études d'analyse fonctionnelle et de maintenabilité sont également à mener d'une manière identique à celles développées dans le monde industriel. Ainsi pourront être mis en évidence les contraintes résultant du fonctionnement de ces bassins, contraintes qui influeront en retour sur la conception de l'ouvrage.

Cette démarche s'intègre également parfaitement sur le plan économique car elle permet de formaliser la relation coût d'investissement/coût d'exploitation en liaison avec la relation conception/entretien.

\section{Bibliographie}

[1] Restauration et Gestion d'un espace vert. Université de Bordeaux I, UER de Biologie, octobre 1987.

[2] Les bassins d'orage sur les réseaux d'assainissement, Agence de l'Eau Artois-Picardie, mars 1988.

[3] Les bassins de pollution : problématique, conception, réalisation. Pierre Giersch, DDAF Bas-Rhin, novembre 1989.

[4] Etude d'une retenue d'eaux pluviales, Laurence Burgaud, Rapport de stage ISIM, septembre 1991. 